Research Article

\title{
Scattering Characteristics of Targets above a Rough Surface in SAR Images
}

\author{
Bo Zhao and Tie Jun Cui \\ Institute of Target Characteristics and Identification and the State Key Laboratory of Millimeter Waves, \\ Department of Radio Engineering, Southeast University, Nanjing 210096, China \\ Correspondence should be addressed to Bo Zhao; bozhao_seu@163.com
}

Received 7 May 2012; Revised 21 November 2012; Accepted 20 December 2012

Academic Editor: Saba Mudaliar

Copyright (C) 2013 B. Zhao and T. J. Cui. This is an open access article distributed under the Creative Commons Attribution License, which permits unrestricted use, distribution, and reproduction in any medium, provided the original work is properly cited.

The modeling, simulation, and analysis of target characteristics are essential to a synthetic aperture radar (SAR) image-based autotarget recognition (ATR) system. The coupling effect between targets and rough surface is also important to the electromagnetic scattering and remote sensing. In this work, the simulations to SAR images of targets above a finite rough surface have been investigated. The effect of rough surface on the target characteristics, or the coupling between the rough surface and targets, is analyzed in details by observing changes of locations and intensities of scattering centers in the SAR images. The SAR images are obtained by taking two-dimensional inverse fast Fourier transforms (FFTs) of the scattered fields, which are computed by the combined high-frequency method of shooting and bouncing ray (SBR) and truncated-wedge incremental-length diffraction coefficients (TW-ILDCs). Simulated results of SAR images for complicated targets above a rough surface are given under the 0.25 $\times 0.25 \mathrm{~m}^{2}$ resolution at the $\mathrm{X}$ band, in which the coupling effect between targets and rough surface has been studied in details.

\section{Introduction}

The classification to the region of interests (ROIs) or the scattering features in synthetic aperture radar (SAR) images is the final and most important stage in a SAR-image-based autotarget recognition (ATR) system. The simulation of SAR images has significant importance to the image understanding and target recognition. Compared to the inverse SAR (ISAR) images of targets in the free space such as airbornes, the simulations of SAR images to various vehicles on the ground or ships on the sea are much more complicated and difficult. In such simulations, the effects of ground or sea surface to the scattering from targets need to be considered, which is important to the real ATR system.

In the past decades, the approaches on how to take into account the coupling between the ground or sea surface and targets have been extensively studied. Johnson calculated the scattering of dielectric objects in a half space using the four-path method [1]. Guan et al. computed the scattered fields of a perfectly electrical conducting (PEC) target above a finite PEC rough surface using the method of moment (MoM) and half-space Green's function [2]. Xpatch could be used to calculate the scattering by objects-either PEC or dielectric-above a dielectric rough surface using the high-frequency (HF) methods and generate the SAR images rapidly [3-5]. However, the parameters of the ground or sea surface, including the dielectric constant, and electric conductivity could not be enumerated. On the SAR image simulations, Franceschetti et al. developed a method to produce SAR raw signals, including the stripe and spotlight mode based on the Kirchhoff approximation (KA) method [6]. Depending on this method, the ground or sea surface has been modeled as a rough surface divided by quadrilateral meshes, and the scattering from every mesh conforms to the Gaussian distribution. Xu and Jin generated a set of SAR images of wider scene with different vegetation and buildings using a mapping and projection algorithm (MPA) [7]. 
Under the point-target hypothesis, a real target consists of many scattering points or light spots in its SAR image. However, the light spots are not always appearing inside the SAR image, while they would change with the incident frequency and attitude angle of the target. Another obvious feature in the SAR image is that a shadow exists on the back to the radar sight. The shape of the radar shadow is similar to the shadow when illuminated by light but is not equal to that. The radar shadow is fuzzier than lighting shadow because of the coherent imaging mechanism and the resolution ratio. In the ATR system, the frequently used features include the location and intensity of the scattering centers, the contour of the target, and the shadow. However, the above features of a target alone will be different to those of the target above the ground or sea surface, while the latter will be used in the real ATR systems.

Hence the coupling between the surface and target must be considered when studying the target features in SAR images. In this paper, the frequency-domain scattered fields of targets above a rough surface are computed using the shooting and bouncing ray (SBR) technique and the truncated-wedge incremental-length diffraction coefficient (TW-ILDC) method. The SAR images of targets are then calculated by taking a two-dimensional (2D) IFFT on the scattered fields, in which the coupling effects have been investigated and interpreted in details. When taking IFFT, the resampling and window function methods are used to preprocess the raw frequency-domain data.

The paper is organized as follows. The SBR technique and TW-ILDC method are introduced in Section 2.1, and the simulations of SAR images are investigated in Section 2.2. Numerical examples are presented in Section 3, which include the targets in free space, the rough surface itself, and the coupling between targets and rough surface. Finally, a conclusion is made in Section 4 to illustrate the importance of the simulations in the real ATR system.

\section{SAR-Image Simulations to Targets above a Rough Surface}

2.1. Computation of Scattered Fields. After developed by Ling et al. [8] to calculate the scattering of cavity, the SBR technique had been used to solve the scattering problems for large electrical-size targets successfully. Xpatch is a wellknown software combining SBR and other effective methods to calculate the radar cross sections (RCSs), SAR images, and ISAR images of targets.

The main idea of SBR is to use a dense grid of shooting rays as the incident field, then calculate the multiple reflections of every shooting ray by the geometrical optics (GO) method when the ray reacts to the target, and finally to compute the scattered fields using the physical optics (PO) method when the ray escapes from the target. The most time consuming part in SBR is not the electromagnetic (EM) computing but the ray tracing, which could be accelerated by means of parallel methods or GPU techniques. The disadvantage of SBR is that it does not include the diffraction and creeping waves, which would influence the accuracy of computation. The equivalent PO current on the surface of a PEC target is written as

$$
\mathbf{J}_{s}=2 \delta \widehat{n}\left(\mathbf{r}^{\prime}\right) \times \mathbf{H}^{i}\left(\mathbf{r}^{\prime}\right)
$$

in which the coefficient $\delta$ accounts for the shadowing effects, and $\hat{n}$ is the outward unit normal vector to the illuminated object surface at $\mathbf{r}^{\prime}$. We could calculate the scattering electrical field by

$$
\mathbf{E}_{s}(\mathbf{r})=-j \omega \mu_{0} \iint \mathbf{J}\left(\mathbf{r}^{\prime}\right) G\left(\left|\mathbf{r}-\mathbf{r}^{\prime}\right|\right) d^{2} r^{\prime},
$$

where $G\left(\left|\mathbf{r}-\mathbf{r}^{\prime}\right|\right)$ is the scaler Green's function in free space

$$
\mathbf{G}\left(\left|\mathbf{r}-\mathbf{r}^{\prime}\right|\right)=\frac{e^{-j k_{0} r}}{4 \pi r} e^{-j \mathbf{k}_{s} \cdot \mathbf{r}^{\prime}}
$$

To improve the accuracy of the SBR technique, the TWILDC method has been considered to include the diffractions from finite sized wedge faces $[9,10]$. The TW-ILDC method has been confirmed to be useful in improving the accuracy, especially for the complicated targets. The detailed derivation of this method and the numerical results have been presented in $[11,12]$. The total scattered field is the superposition of the SBR field and the diffraction field as follows:

$$
\mathbf{E}_{s}=\mathbf{E}_{s}^{\mathrm{PO}}+\mathbf{E}_{s}^{\text {Diffraction }}
$$

In general, the frequency-domain scattered field could be computed by the combined SBR and TW-ILDC methods [11].

2.2. The SAR-Image Generation. It has been proved that the SAR image could be generated from the frequencydomain scattered fields using the 2D-IFFT algorithm [13]. The incident magnetic field is obtained by $\mathbf{H}_{i}(\mathbf{r})=(1 / \omega \mu) \mathbf{k}_{i} \times$ $\mathbf{E}_{i}$ from the specific incident electric field. Then the scattered field is written as

$$
\mathbf{E}_{s}(\mathbf{r})=-j k_{0} E_{0} \iint 2 \widehat{n}(\mathbf{r}) \times\left(\widehat{k}_{i} \times \hat{t}\right) e^{-j \mathbf{k}_{i} \cdot \mathbf{r}^{\prime}} G\left(\left|\mathbf{r}-\mathbf{r}^{\prime}\right|\right) d^{2} r^{\prime}
$$

We suppose that the $\widehat{p}$ component of the scattering field is received, and define a shape function $O\left(\mathbf{r}^{\prime}\right)$. Then the $\widehat{p}$ component of the scattered field is written as

$$
\widehat{p} \cdot \mathbf{E}_{s}(\mathbf{r})=-j k_{0} E_{0} \frac{e^{-j k_{0} r}}{4 \pi r} \iiint_{-\infty}^{+\infty} O\left(\mathbf{r}^{\prime}\right) e^{-2 j \mathbf{k}_{i} \cdot \mathbf{r}^{\prime}} d^{3} r^{\prime},
$$

from which the relationship between the scattered field and the Fourier transform of the shape function is obtained as follows:

$$
\widehat{p} \cdot \mathbf{E}_{s}(\mathbf{r})=C \widetilde{O}\left(\mathbf{k}_{s}-\mathbf{k}_{i}\right),
$$

in which $\widetilde{O}(\mathbf{r})$ is the Fourier transform of the shape function

$$
\widetilde{O}(\mathbf{k})=\iiint_{-\infty}^{+\infty} O\left(\mathbf{r}^{\prime}\right) e^{j \mathbf{k} \cdot \mathbf{r}^{\prime}} d^{3} r^{\prime}
$$


That is to say that the shape of the target could be obtained from the scattered field by using IFFT [13].

In this paper, the 2D SAR image will be considered, which is the projection of the shape function on the imaging plane, the $x-y$ plane. Hence the relationship between the 2D SAR image and the shape function is

$$
O\left(k_{x}, k_{y}\right)=\iint_{-\infty}^{\infty} O_{\text {proj }}(x, y) e^{j k_{x} x+j k_{y} y} d x d y .
$$

From the above equations, it is clear that we could obtain the SAR images from the scattered fields simulated by the SBR and TW-ILDC methods. First, a set of scattered field data are simulated. Then a similar procedure is conducted to the PFA step in the spotlight SAR imaging, which takes an interpolation on the data before performing IFFT. The window function method is applied before IFFT to reduce the Gibbs' effect [14]. To obtain a high-resolution SAR image, a set of wideband and wide-azimuth data are needed. The resolution in the range and azimuth, $\rho_{r}$ and $\rho_{a}$, is to be determined as

$$
\rho_{r}=\frac{c}{2 B}, \quad \rho_{a}=\frac{c}{4 f_{c} \sin (\Delta \phi) / 2},
$$

where $c$ is the light velocity, $B$ is the bandwidth of the electromagnetic wave, $f_{c}$ is the central frequency, and $\Delta \phi$ is the rotation angle of SAR.

The simulated data are distributed over a sector region in the wave number domain (WND) on the $k_{x}-k_{y}$ plane. Although the distribution region is similar to a rectangle, it is still different (the azimuth is large). Hence a sector-torectangle interpolation, which is called as resampling in PFA, is needed before IFFT. Once the resolutions are confirmed, the resampling numbers could be computed as follows:

$$
N_{r}=\frac{L_{\mathrm{max}}}{\rho_{r}}, \quad N_{a}=\frac{L_{\mathrm{max}}}{\rho_{a}},
$$

where $L_{\max }$ is the maximum length of the simulation scene. On the other hand, to reduce the Gibbs' effect, we use a $2 \mathrm{D}$ Taylor window function on the resampled data in the $k$ domain [14].

\section{Numerical Examples}

The main purpose of this paper is to illustrate the effect of a rough surface to SAR images of targets located above the rough surface. The Gaussian distribution rough surfaces has been widely used in the scattering research, which is chosen as ground model. Because the SBR method is used to calculate scatter field of models, the rough surface is also set as PEC objects. The rough surface is described as the Gaussian spectrum by

$$
W\left(k_{x}, k_{y}\right)=\frac{l_{x} l_{y} h^{2}}{4 \pi} \exp \left[\frac{l_{x}^{2} k_{x}^{2}+l_{y}^{2} k_{y}^{2}}{4}\right],
$$

in which $l_{x}$ and $l_{y}$ are the correlation lengths in $x$ - and $y$ directions, respectively, and $h$ is the rms height of the rough surface. The generation progress has been shown in [15]. In the SBR method, the simulation time is related to the size and mesh density of the targets. Hence an infinite rough surface is impossible for simulations and we have to choose a finite-sized rough surface. The model should be static PEC or dielectric coated electrically large ground targets and the size of targets should be larger than the minimum resolution cell in the image. It also could be meshed before calculation. The incident parameters are defined in Figure 1(a) and the size of rough surface is set in a minimum length as shown in Figure 1(b) because it could contain the shadow area which could be an important feature in an ATR system.

Here are the explanation of the multiple scattering routes on the rough surface. In Figure 1(b), we could see that there are several scattering routes to contribute to the back scattering, which are

(1) the direct reflection by the rough surface;

(2) the direct reflection by the target;

(3) multiple scattering from the target to rough surface;

(4) multiple scattering from the rough surface to target.

There are also other multiple scattering that could be ignored. Each route could contain numbers of reflections during SBR method [8]. In this paper, the number of reflections could be 2 or 3 according to different models and could satisfy the calculation accuracy $[11,12]$.

In the first example, the effect of a rough surface to targets is investigated through the simulation of a SLICY-like target above a rough surface which is shown in Figure 2. SLICY is a vehicle-size target which includes canonical shapes and cylindrical surfaces. The forward direction is looking into the trihedral corner, which we call the front of the target. The maximum dimensions of SLICY-like target shown in 2 are $81.5 \lambda$ in width, $125.8 \lambda$ in length, and $64.7 \lambda$ in height. In the following simulations, all the rough surfaces are discretized by flat triangles whose maximum edge length is $3 \lambda$ ( $\lambda$ is the wavelength in free space).

The target is located above a Gaussian rough surface with $l_{x}=l_{y}=15 \lambda$ and $h=2 \lambda$. The horizontal dimensions of the rough surface are set as $350 \lambda \times 350 \lambda$. The plane waves are incident from the direction of $\theta=75^{\circ}$ and $\phi=\left(0^{\circ}, 15^{\circ}, 30^{\circ}, 45^{\circ}, 60^{\circ}, 75^{\circ}, 90^{\circ}\right)$, respectively, the central frequency is $10 \mathrm{GHz}$, and the bandwidth is $1.5 \mathrm{GHz}$. Here, the incident direction implies the central direction of SAR simulation because $N_{a}$ directions in the range of $\Delta \phi$ are required. From (10) we can calculate that the range resolution is $0.1 \mathrm{~m}$. We also suppose that the azimuth resolution is equal to the range resolution, so that $\Delta \phi$ is $4.29546^{\circ}$.

Figure 3 shows the SAR image results of SLICY-like target on the Gaussian rough surface for the $\mathrm{HH}$ polarizations where $y$-axis represents range direction and $x$-axis represents cross-range direction. The horizontal streak in Figures 3(a), $3(\mathrm{~b}), 3(\mathrm{~g})$, and $3(\mathrm{~h})$ is mainly caused by the Gibbs' effect during IFFT, especially in Figures 3(a) and 3(g). An obvious feature of Gibbs' effect in SAR image is sidelobes next to the strong scattering points. Results of VV have similar characteristics, actually. To illustrate the effect of scattering from the rough surface, the corresponding SAR images of 


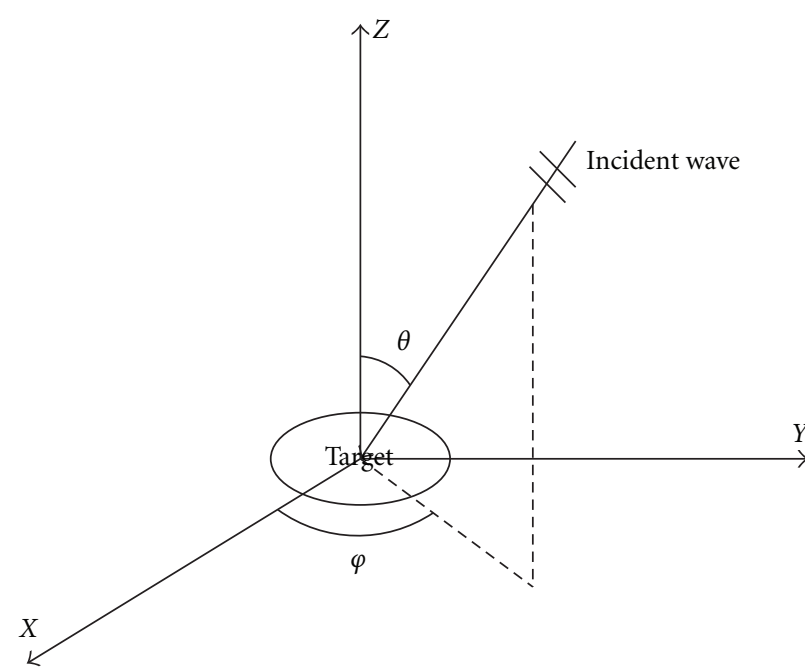

(a)

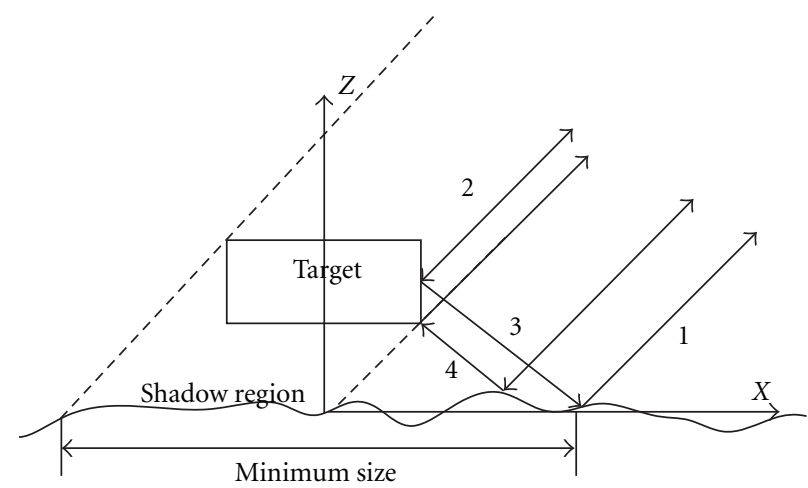

(b)

FIGURE 1: The incident parameters definition and the minimum size of a rough surface.

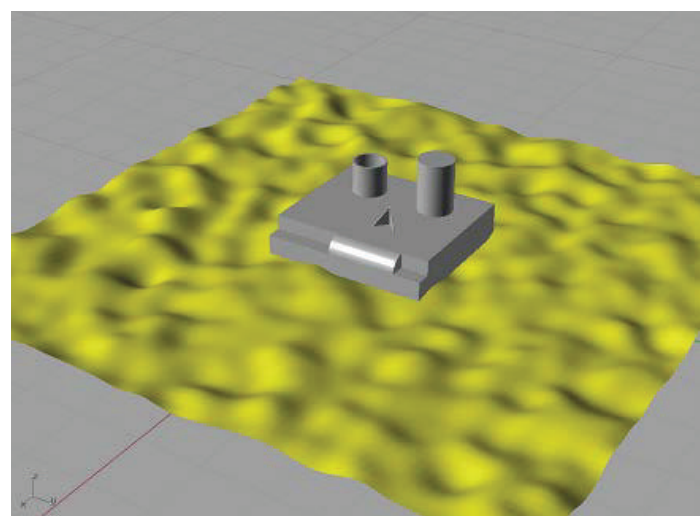

Figure 2: The geometrical model of a SLICY-like target above a Gaussian rough surface.

the target in free space are also simulated. Because the main strong scattering effects are brought by the first three reflections, the multiple reflections more than 4 are ignored. Limited by space, only the results of $\phi=\left(0^{\circ}, 30^{\circ}, 60^{\circ}, 90^{\circ}\right)$ are demonstrated here, which are clear enough to show the rough-surface effect.

The strong scattering points in SAR images are generated mainly by the dihedral angles and trihedral angles on the target. When SLICY is placed above a rough surface, there is a local "dihedral angles" which supply multireflections constructed by the bottom of target and rough surface so that there are obvious differences between the results of free space and above the rough surface, as illustrated in Figures 3(a), 3(b), 3(g), and 3(h). Although the "dihedral angles" are different to those constructed by the target and a PEC plane, the construction still have similar multireflection route during ray tracing in SBR method especially enhancing the backscattering. Meanwhile, in Figures 3(c), 3(d), 3(e), and $3(\mathrm{f})$, although there are multireflection constructions at the bottom of the target, the second reflection will not return back because the direction of the reflection is different to the backscattering. Comparing to the multireflection part, the backscattering of the rough surface is so small that it could not be displayed clearly in the images.

To compare the differences of the results in free space and above the rough surface, we calculate the correlation coefficient of the two images in all of the 7 directions. The correlation coefficient is defined as

$$
\text { corrcoef }=\frac{E(A B)-E(A) E(B)}{\sqrt{E\left(A^{2}\right)-E^{2}(A)} \sqrt{E\left(B^{2}\right)-E^{2}(B)}},
$$

where $A$ and $B$ are the two image matrices. The corrcoef is close to 1 when $A$ and $B$ are similar to each other, and if $A=B$ then corrcoef $=1$. The curve of the correlation coefficient is shown in Figure 4.

From Figure 4 we can see that the correlation coefficients are close to 1 except two special directions at $\phi=0^{\circ}$ and $90^{\circ}$. Results in Figures 3 and 4 illustrate that the rough surface always impact the SAR image results by changing the local consistence of reflectors. However, the other reflectors located on the plane of the SLICY-like target are not influenced by the rough surface so that the correlation coefficients at directions of $\phi=\left(15^{\circ}, 30^{\circ}, 45^{\circ}, 60^{\circ}, 75^{\circ}\right), \theta=75^{\circ}$ are close to 1 .

In an SAR-based ATR system, the strong scattering points are the most important features that would be used during the matching procedure. Next we present simulation results of a complicated target, a model of tank, to illustrate the SAR images in free space and above a rough surface and the changes of amplitudes and locations of scattering points when the target is above different rough surfaces. There are also tank results that could be obtained in $[4,5]$. The size of tank model is $295 \lambda$ in length, $107 \lambda$ in width, and $73 \lambda$ in height. The three-dimensional (3D) model of the tank above a Gaussian rough surface is shown in Figure 5. 


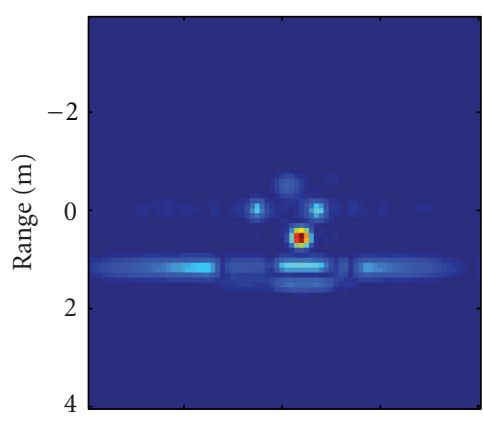

(a) Cross-range (m)

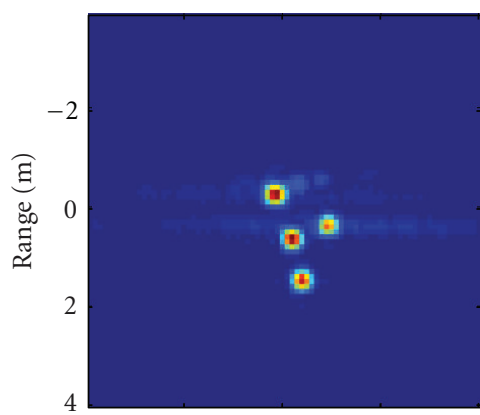

(c) Cross-range $(\mathrm{m})$

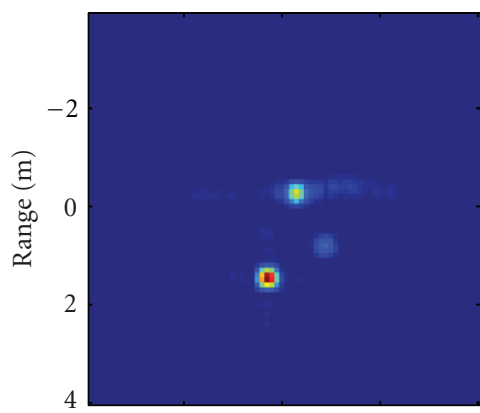

(e) Cross-range $(\mathrm{m})$

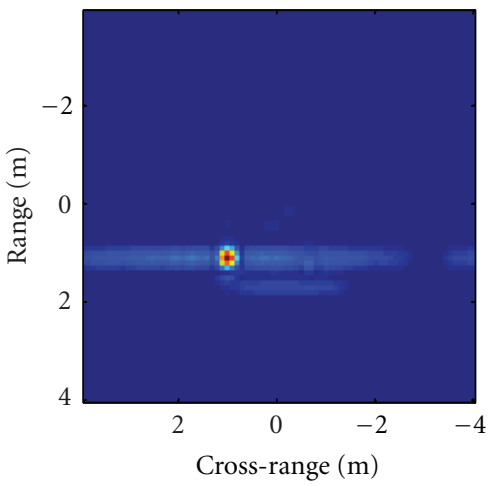

(g)

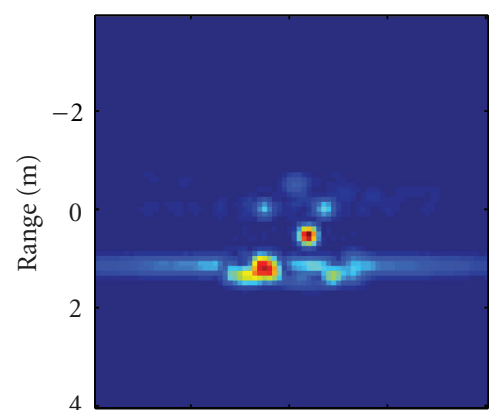

(b) Cross-range (m)

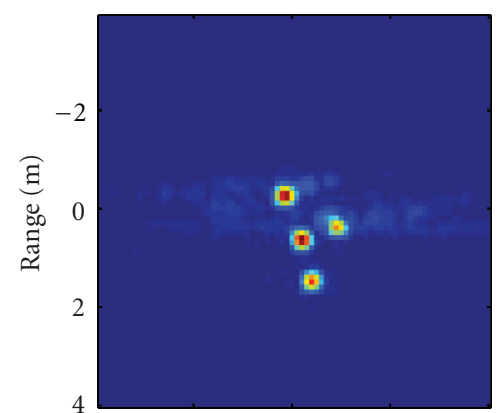

(d) Cross-range (m)

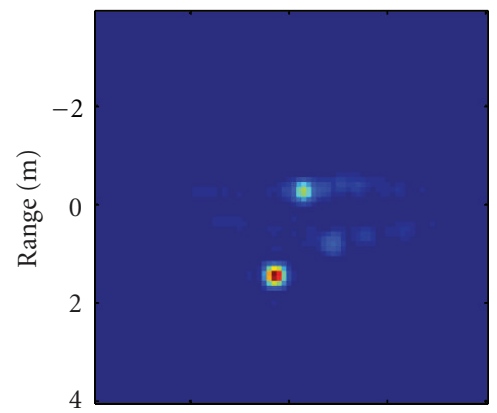

(f) Cross-range $(\mathrm{m})$

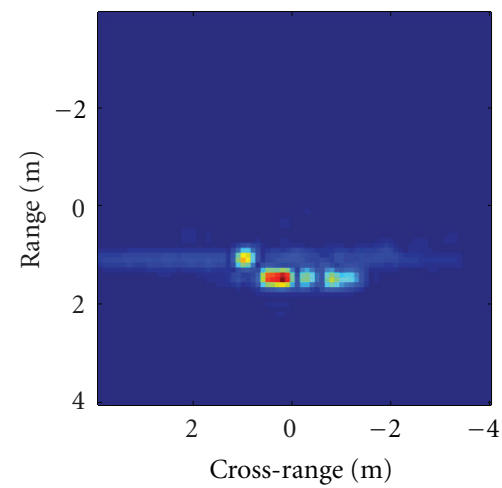

(h)

FIGURE 3: The 2D HH-polarization SAR images of the SLICY-like target in free space and above a Gaussian rough surface, in which the incident direction is $\left(\theta=75^{\circ}\right.$ and $\left.\phi=\left[0^{\circ}, 30^{\circ}, 60^{\circ}, 90^{\circ}\right]\right)$, the resolution is $0.1 \mathrm{~m}$, and the maximum reflection number is 3. (a) The SAR image of target in free space at the azimuth of $\phi=0^{\circ}$. (b) The SAR image of target above a Gaussian rough surface at the azimuth of $\phi=0^{\circ}$. (c) The SAR image of target in free space at the azimuth of $\phi=30^{\circ}$. (d) The SAR image of target above a Gaussian rough surface at the azimuth of $\phi=30^{\circ}$. (e) The SAR image of target in free space at the azimuth of $\phi=60^{\circ}$. (f) The SAR image of target above a Gaussian rough surface at the azimuth of $\phi=60^{\circ}$. (g) The SAR image of target in free space at the azimuth of $\phi=90^{\circ}$. (h) The SAR image of target above a Gaussian rough surface at the azimuth of $\phi=90^{\circ}$. 


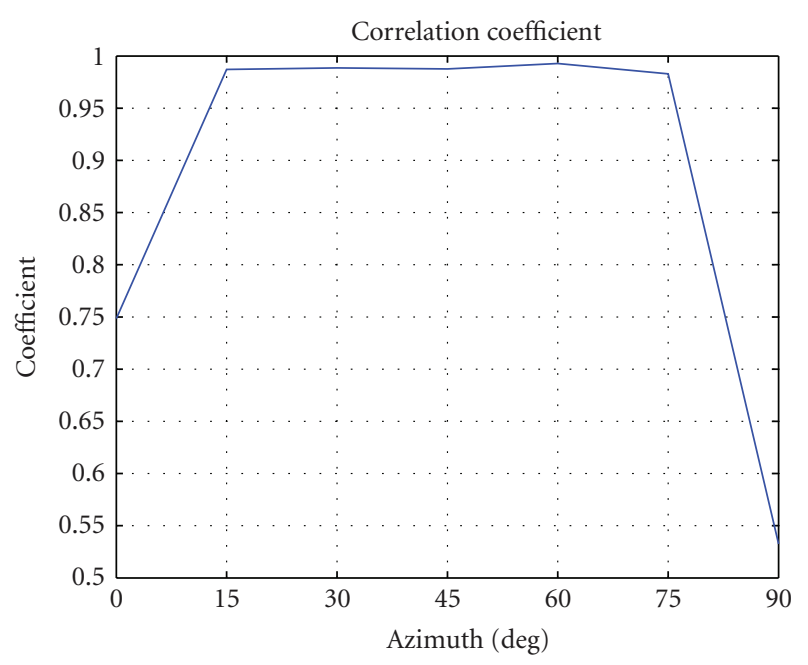

FIGURE 4: The correlation coefficients of the SAR images of the SLICY-like target in free space and above a rough surface at 7 azimuth directions.

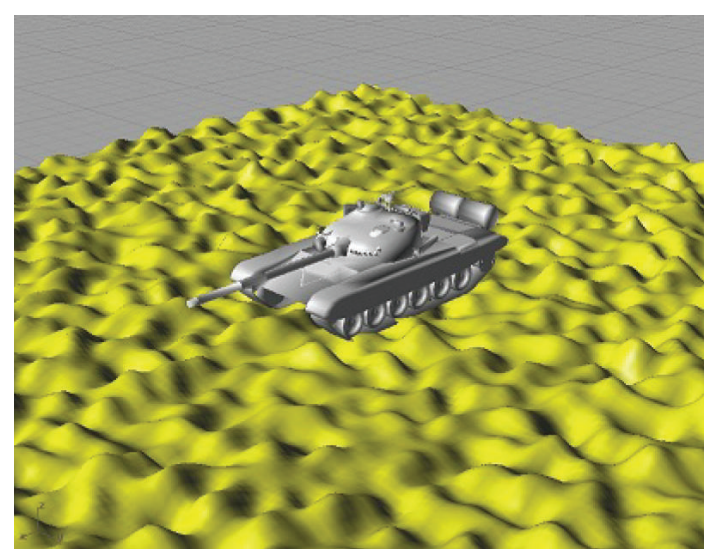

Figure 5: The 3D geometrical model of a tank above a Gaussian rough surface.

Firstly, we consider the tank model above different Gaussian rough surfaces. Generally, the coupling is weak for the parts of rough surfaces which are far from the target because small quantity of rays could reach the target. It is important to confirm the size of the Gaussian rough surface. If the size of the rough surface is small, the accuracy of the scattering effect could not be guaranteed; if the size is very large, however, huge amount of simulation time would be required. Here, we consider two sizes of rough surfaces, which are $1000 \lambda \times 1000 \lambda$ and $1250 \lambda \times 1250 \lambda$, respectively. The correlation length and height of the two rough surfaces are both $l_{x}=l_{y}=15 \lambda$ and $h=5 \lambda$. The direction of the tank gun barrel is set as $0^{\circ}$ azimuth direction, and the three incident directions are set as $\theta=75^{\circ}$ and $\phi=\left[30^{\circ}, 45^{\circ}, 60^{\circ}\right]$. The central frequency of the incident $\mathrm{HH}$-polarization plane wave is $9.5 \mathrm{GHz}$, and the bandwidth is $0.6 \mathrm{GHz}$. The azimuth range is $3.61^{\circ}$. According to (10), the resolutions of the range and cross-range are $\rho_{r}=\rho_{a}=0.25 \mathrm{~m}$.
Figure 6 shows the SAR image results of the tank model above different Gaussian rough surfaces. From top to bottom, each row means different imaging azimuth directions of the target, which are $30^{\circ}, 45^{\circ}$ and $60^{\circ}$, respectively. The left column of Figure 6 is the result with smaller rough surface, and the right column is the result with larger rough surface. The display ranges in the SAR images are the same, but are different from those when simulating since they need different samples to satisfy the Nyquist's sample law. The images are zoomed in to fit the size of tank model to be displayed more clearly. The grounds are generated randomly so that the noise is different.

From Figure 6 we can see that the brightest pixels are nearly the same when the tank target is above different-size rough surfaces. In the azimuth of $30^{\circ}$, the brightest pixels represent the tank wheels, and the next bright pixels located in the right of the brightest ones indicate the oil drums and hatch, which are nearly the same in the two SAR images, as shown in Figures 6(a) and 6(b). The weak scattering points such as the front cover and gun barrel are different in two images because the scattering of these parts is so weak that could be easily submerged by the scattering of rough surfaces. In the azimuth of $45^{\circ}$, the brightest pixels represent the wheels and hatch part of the tank, and the next bright pixels are located in the two points of front cover on the right of the hatch. These features are nearly the same in the two SAR images demonstrated in Figures 6(c) and 6(d), but a tiny difference exists in the gun-barrel scattering point. It is brighter in Figure 6(c).

For the case of $60^{\circ}$ azimuth angle, the SAR image results in such two situations have significant difference. This is because the energy is not concentrated to a few strongscattering points but distributed in many weaker pixels. Hence the scattering by the rough surface could influent the amplitudes and locations of these pixels significantly. There are several obvious differences in the two SAR images, as illustrated in Figures 6(e) and 6(f). First of all, the point located at the back cover appears in Figure 6(f) but disappears in Figure 6(e). Secondly, the two strong scattering points generated by the hatch and toolbox are clearer in Figure 6(f). Regardless of such differences, the distributions of strong scattering points in such two images are basically similar to satisfy the requirement of an ATR system.

Figure 7 shows another SAR image results of the tank model above different rough surfaces, in which the incident angle is $\left(\theta=75^{\circ}, \phi=45^{\circ}\right)$. The sizes of rough surfaces are $800 \lambda \times 800 \lambda, 933 \lambda \times 933 \lambda, 1067 \lambda \times 1067 \lambda, 1200 \lambda \times 1200 \lambda$, and $1333 \lambda \times 1333 \lambda$, respectively. Other parameters are the same as those in Figure 6.

From Figure 7, we can see that the five figures are nearly the same, which means that the coupling between the tank model and rough surface is only concentrated around the tank model. Hence it would be accurate enough if the size of the rough surface is set to $1000 \lambda \times 1000 \lambda$.

In the above examples, the tank target and the rough surface are regarded as a combined system in the numerical simulations. To investigate the coupling effect of rough surface, several "superposed" SAR images are generated 


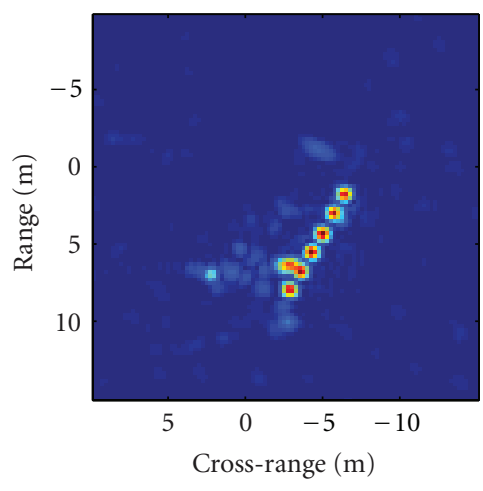

(a)

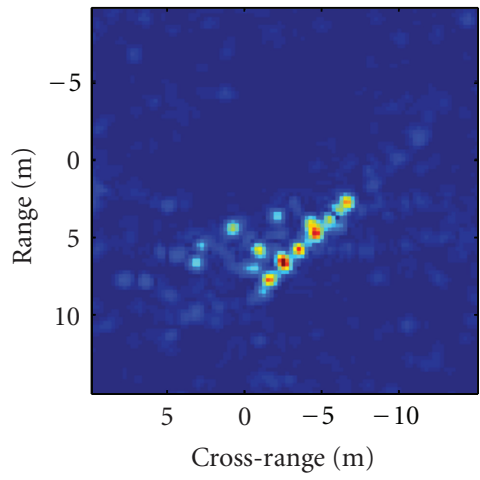

(c)

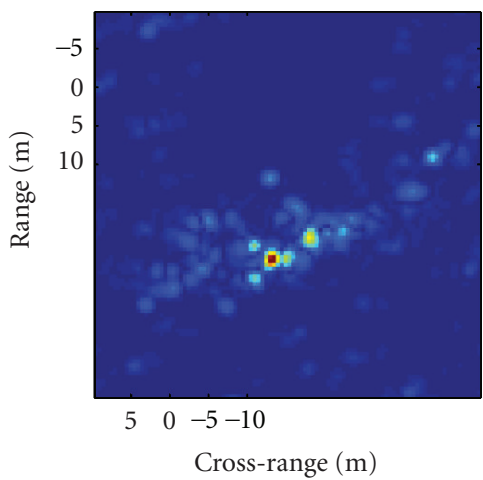

(e)

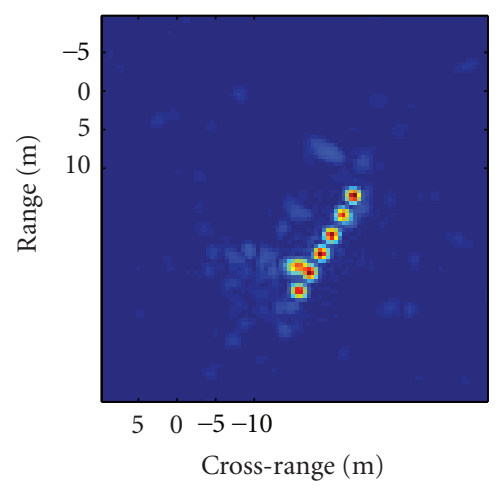

(b)

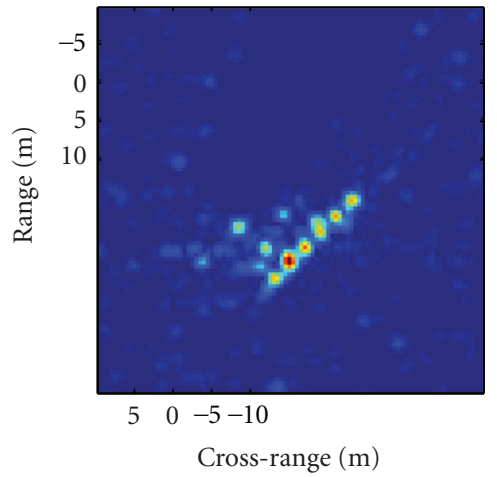

(d)

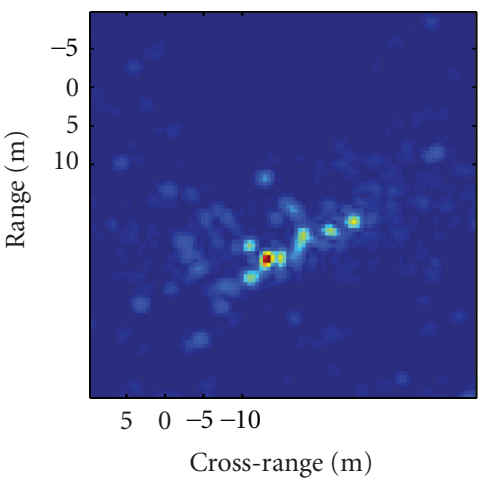

(f)

FIGURE 6: The 2D HH-polarization SAR images of the tank target above Gaussian rough surfaces with different sizes, in which the incident angle is $\left(\theta=75^{\circ}, \phi=\left[30^{\circ}, 45^{\circ}, 60^{\circ}\right]\right)$, the resolution is $0.25 \mathrm{~m}$, and the maximum reflection number is 2 . The gun barrel direction is set as $\phi=0^{\circ}$. (a) The SAR image of the tank above the smaller rough surface at azimuth of $30^{\circ}$. (b) The SAR image of the tank above the larger rough surface at azimuth of $30^{\circ}$. (c) The SAR image of the tank above the smaller rough surface at azimuth of $45^{\circ}$. (d) The SAR image of the tank above the larger rough surface at azimuth of $45^{\circ}$. (e) The SAR image of the tank above the smaller rough surface at azimuth of $60^{\circ}$. (f) The SAR image of the tank above the larger rough surface at azimuth of $60^{\circ}$.

by superposing the free-space target images on the roughsurface images. The correlation coefficient is also calculated to illustrate the similarity of the two kinds of images. We present several simulations to illustrate the difference between the tank target in free space and that above a Gaussian rough surface. The size of the rough surface is $1000 \lambda \times 1000 \lambda$, and other parameters are the same as the earlier example. Figure 8 shows the results of "combined" and "superposed" SAR images of the tank target. The left column is the "superposed" SAR images, while the right column is the "combined" SAR images, respectively. From top to bottom, the azimuth directions are $30^{\circ}, 45^{\circ}$, and $60^{\circ}$, respectively.

From Figure 8 we can see that the obvious difference between the two kinds of SAR images is whether a shadow of 


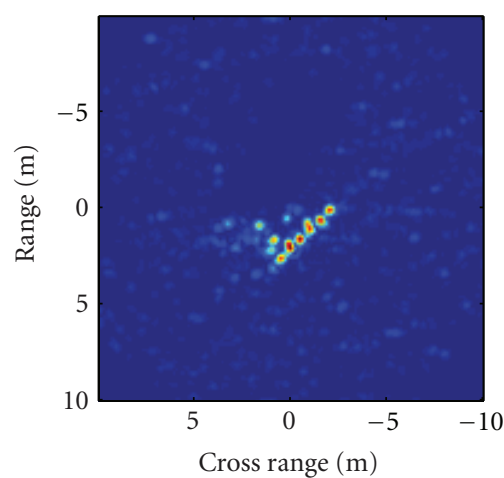

(a)

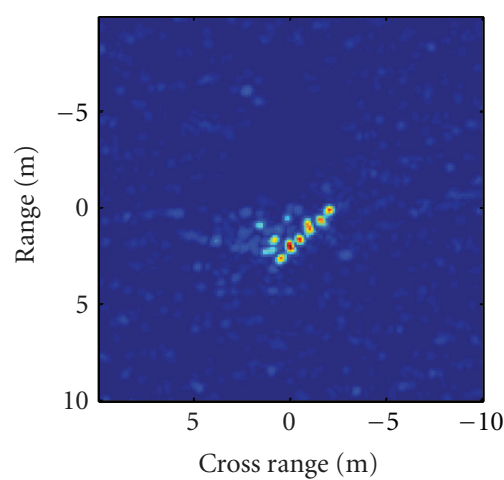

(c)

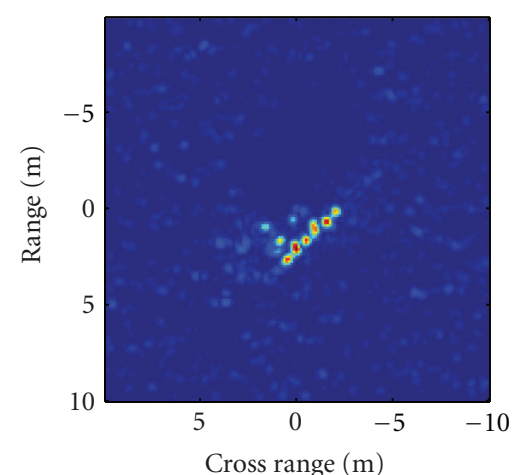

(b)

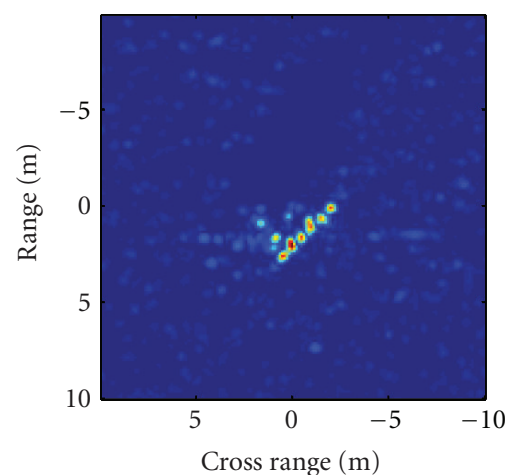

(d)

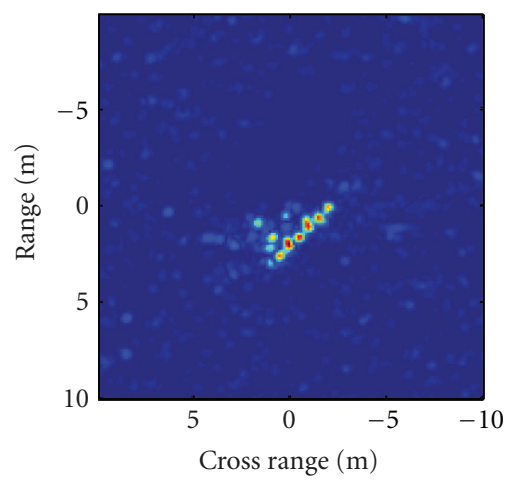

(e)

FIGURE 7: The 2D HH-polarization SAR images of the tank target above Gaussian rough surfaces with different sizes, in which the incident angle is $\left(\theta=75^{\circ}, \phi=45^{\circ}\right)$, other parameters are the same as in Figure 5. (a) The SAR image of the tank above rough surface of $800 \lambda \times 800 \lambda$. (b) The SAR image of the tank above rough surface of $933 \lambda \times 933 \lambda$. (c) The SAR image of the tank above rough surface of $1067 \lambda \times 1067 \lambda$. (d) The SAR image of the tank above rough surface of $1200 \lambda \times 1200 \lambda$. (e) The SAR image of the tank above rough surface of $1333 \lambda \times 1333 \lambda$.

the tank exists or not. The "combined" method is based on the ray-tracing technology, hence the occluded part of the rough surface by the tank could not be illuminated by rays. Besides, there are other differences in which we have more interest. In the azimuth of $30^{\circ}$ shown in Figure $8(\mathrm{~b})$, an extra strong scattering point exists at the right-hand of gun barrel, which disappeared in Figure 8(a). In the azimuth of $45^{\circ}$, the two SAR images have a difference on the scattering points of wheel locations, which implies that the rough surface and the tank confirm a local reflection structure which changes the route of reflected rays. In the azimuth of $60^{\circ}$, the strong scattering points are basically the same, but there is an extra point at $(21,16)$ in Figure $8(\mathrm{f})$. This point is caused by the multiple reflections from the tank to the rough surface, which do not exist in Figure 8(e).

\section{Conclusions}

In this paper, the scattering characteristics of targets above a Gaussian rough surface in SAR images are investigated via 


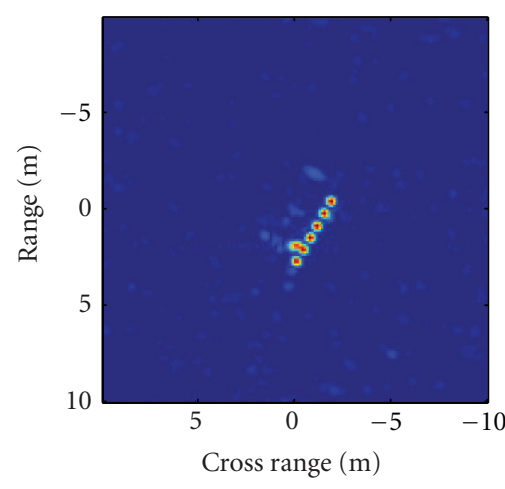

(a)

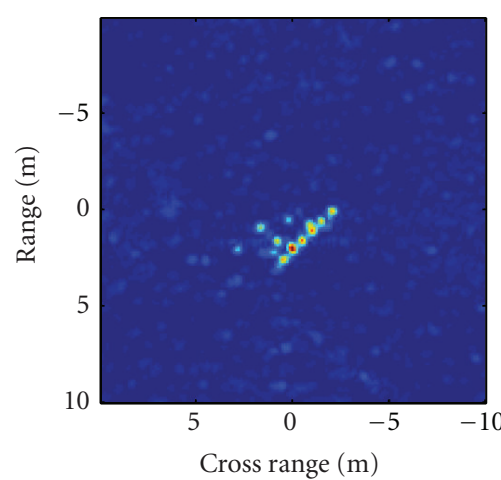

(c)

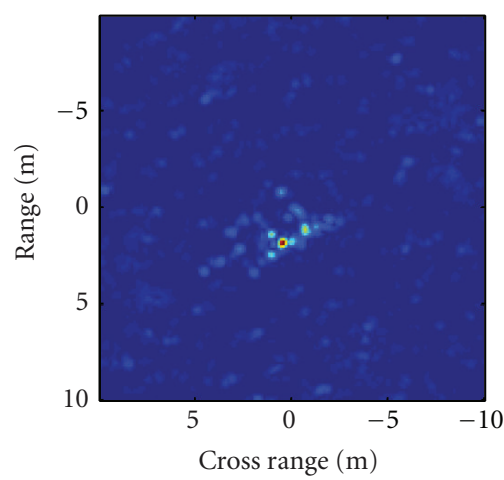

(e)

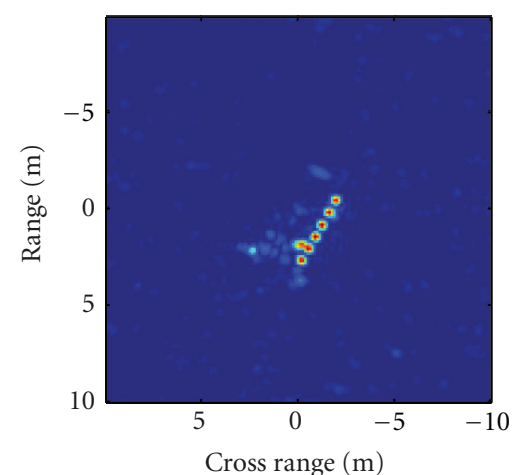

(b)

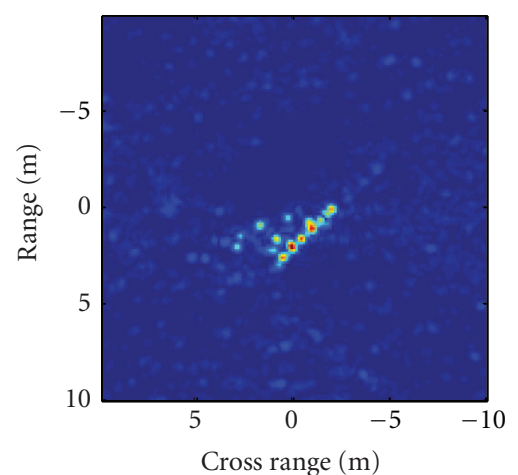

(d)

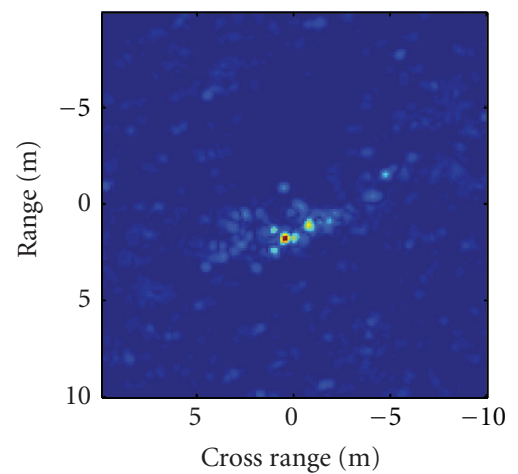

(f)

FIGURE 8: The 2D HH-polarization "superposed" and "combined" SAR images of the tank target and Gaussian rough surface, in which the incident angle is $\left(\theta=75^{\circ}, \phi=\left[30^{\circ}, 45^{\circ}, 60^{\circ}\right]\right)$, the resolution is $0.25 \mathrm{~m}$, and the maximum reflection number is 2 . The gun barrel direction is set as $\phi=0^{\circ}$. (a) The SAR image of the tank in the superposed way at azimuth of $30^{\circ}$. (b) The SAR image of the tank in the combined way at azimuth of $30^{\circ}$. (c) The SAR image of the tank in the superposed way at azimuth of $45^{\circ}$. (d) The SAR image of the tank in the combined way at azimuth of $45^{\circ}$. (e) The SAR image of the tank in the superposed way at azimuth of $60^{\circ}$. (f) The SAR image of the tank in the combined way at azimuth of $60^{\circ}$.

the HF asymptotic method. The presented numerical results demonstrate that some differences exist between the SAR images of target in free space and those above the Gaussian rough surface. This kind of phenomenon is principally caused by the local change of structure consisting of the target and the rough surface. We can use these changes of characteristics to improve the matching stage of a SAR-based ATR system.

\section{References}

[1] J. T. Johnson, "A study of the four-path model for scattering from an object above a half space," Microwave and Optical Technology Letters, vol. 30, no. 2, pp. 130-134, 2001.

[2] B. Guan, J. F. Zhang, X. Y. Zhou, and T. J. Cui, "Electromagnetic scattering from objects above a rough surface using the method 
of moments with halfspace green's function," IEEE Transactions on Geoscience and Remote Sensing, vol. 47, no. 10, pp. 3399-3405, 2009.

[3] D. J. Andersh, S. W. Lee, J. Moore, and D. Sullivan, "Xpatch prediction improvements to support multiple ATR applications," in SPIE Conference on Radar Sensor Technology III, vol. 3395, pp. 108-119, 1998.

[4] W. Coburn, C. Le, C. Kenyon, A. Sullivan, and R. Namburu, "Radar signature modeling for combat systems," in Proceedings of the 2003 User Group Conference, pp. 68-73, 2003.

[5] W. Coburn, C. Le, and W. Spurgeon, "Potential discrepancies in radar signature predictions for ground vehicles," in Proceedings of the 2003 User Group Conference, pp. 45-51, July 2004.

[6] G. Franceschetti, M. Migliaccio, D. Riccio, and G. Schirinzi, "SARAS: a synthetic aperture radar (SAR) raw signal simulator," IEEE Transactions on Geoscience and Remote Sensing, vol. 30, no. 1, pp. 110-123, 1992.

[7] F. Xu and Y. Q. Jin, "Imaging simulation of polarimetric SAR for a comprehensive terrain scene using the mapping and projection algorithm," IEEE Transactions on Geoscience and Remote Sensing, vol. 44, no. 11, pp. 3219-3234, 2006.

[8] H. Ling, R. C. Chou, and S. W. Lee, "Shooting and bouncing rays: calculating the RCS of an arbitrarily shaped cavity," IEEE Transactions on Antennas and Propagation, vol. 37, no. 2, pp. 194-205, 1989.

[9] R. A. Shore and A. D. Yaghjian, "Shadow boundary incremental length diffraction coefficients applied to scattering from 3-D bodies," IEEE Transactions on Antennas and Propagation, vol. 49, no. 2, pp. 200-210, 2001.

[10] P. M. Johansen, "Uniform physical theory of diffraction equivalent edge currents for truncated wedge strips," IEEE Transactions on Antennas and Propagation, vol. 44, no. 7, pp. 989-995, 1996.

[11] Z. Li, T. J. Cui, X. J. Zhong, Y. B. Tao, and H. Lin, "Electromagnetic scattering characteristics of PEC targets in terahertz regime," IEEE Transactions on Advanced Packaging, vol. 41, pp. $1-9,2008$.

[12] Z. Li and T. J. Cui, "High frequency methods for simulation of high resolution imaging in terahertz regime," Journal of Infrared, Millimeter, and Terahertz Waves, vol. 31, no. 3, pp. 349-357, 2010.

[13] R. Bhalla and H. Ling, "Isar image formation using bistatic computed from shooting and bouncing ray technique," Journal of Electromagnetic Waves and Application, vol. 7, no. 9, pp. 1271-1287, 1993.

[14] W. G. Garrara, R. S. Goodman, and R. M. Majewski, Spotlight Synthetic Aperture Radar Signal Processing Algorithms, Artech House, 1993.

[15] E. I. Thorsos, "The validity of the Kirchhoff approximation for rough surface scattering using a Gaussian roughness spectrum," The Journal of the Acoustical Society of America, vol. 83, no. 1, pp. 78-92, 1988. 

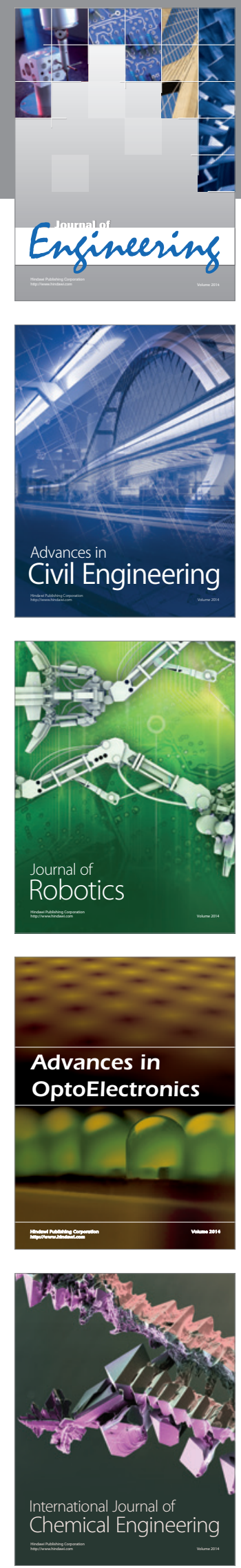

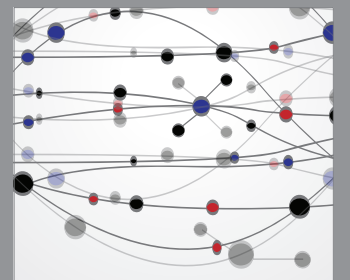

The Scientific World Journal
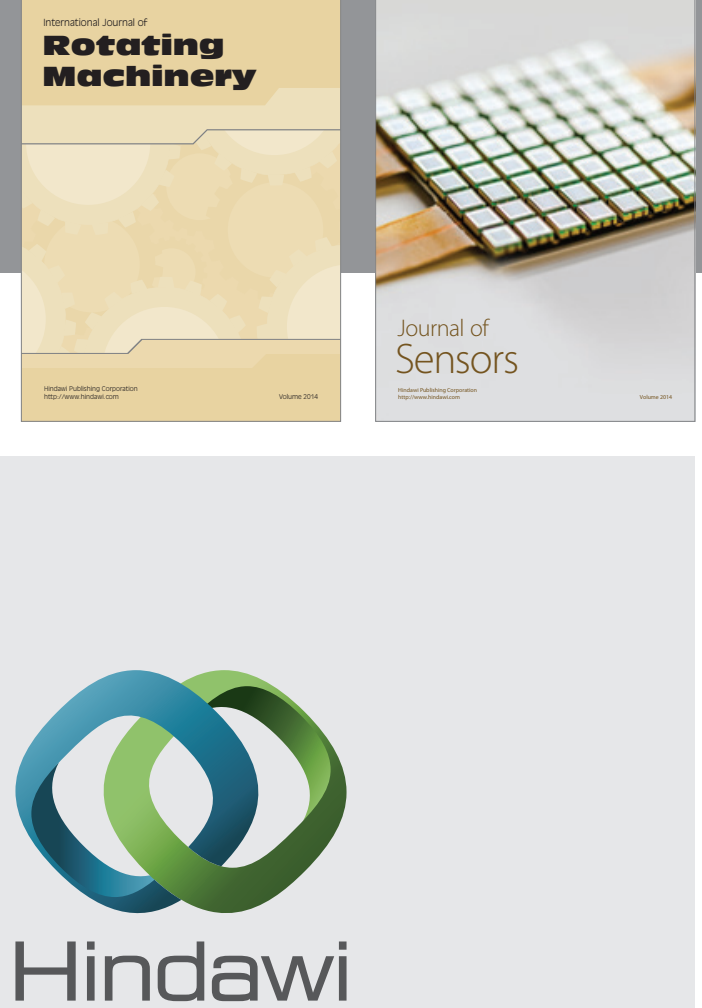

Submit your manuscripts at http://www.hindawi.com
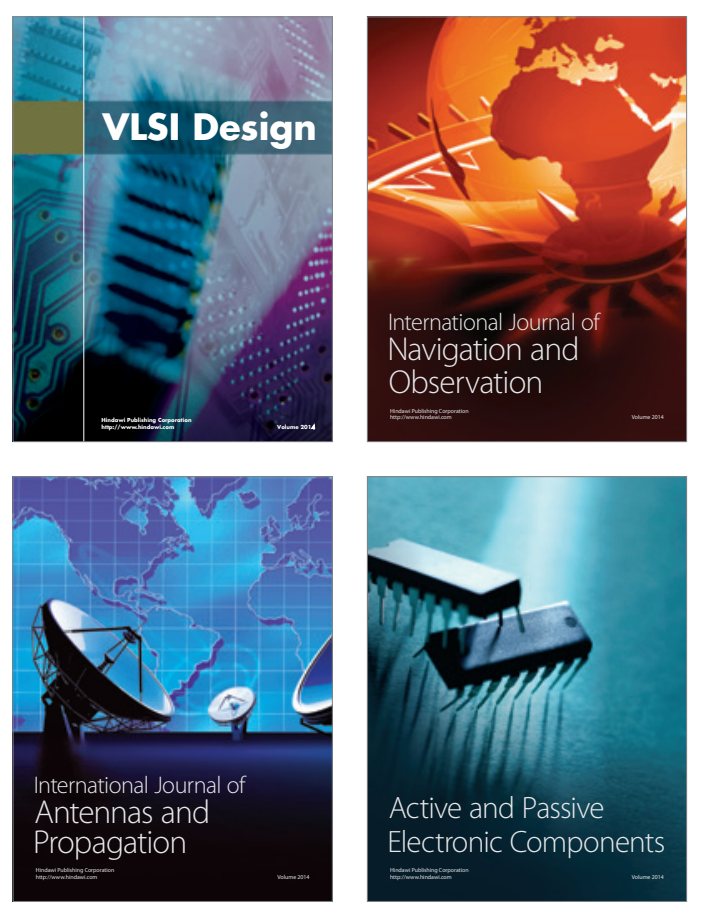
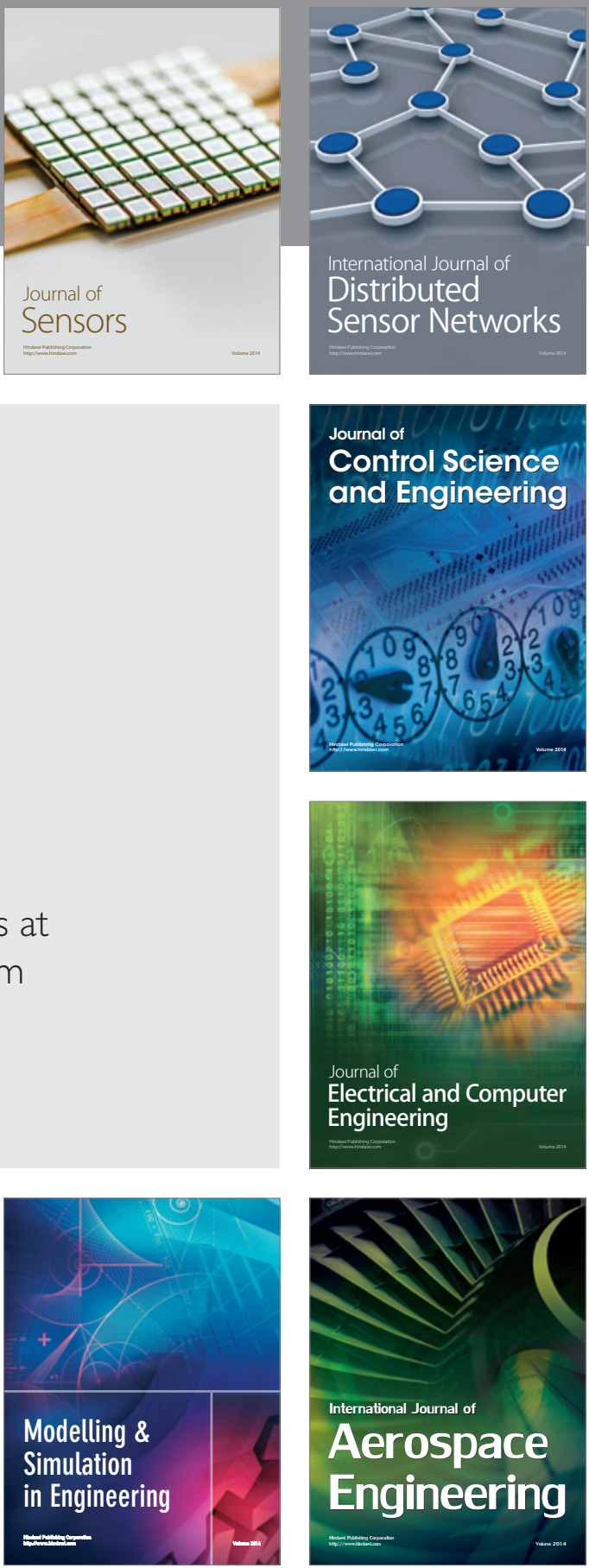

Journal of

Control Science

and Engineering
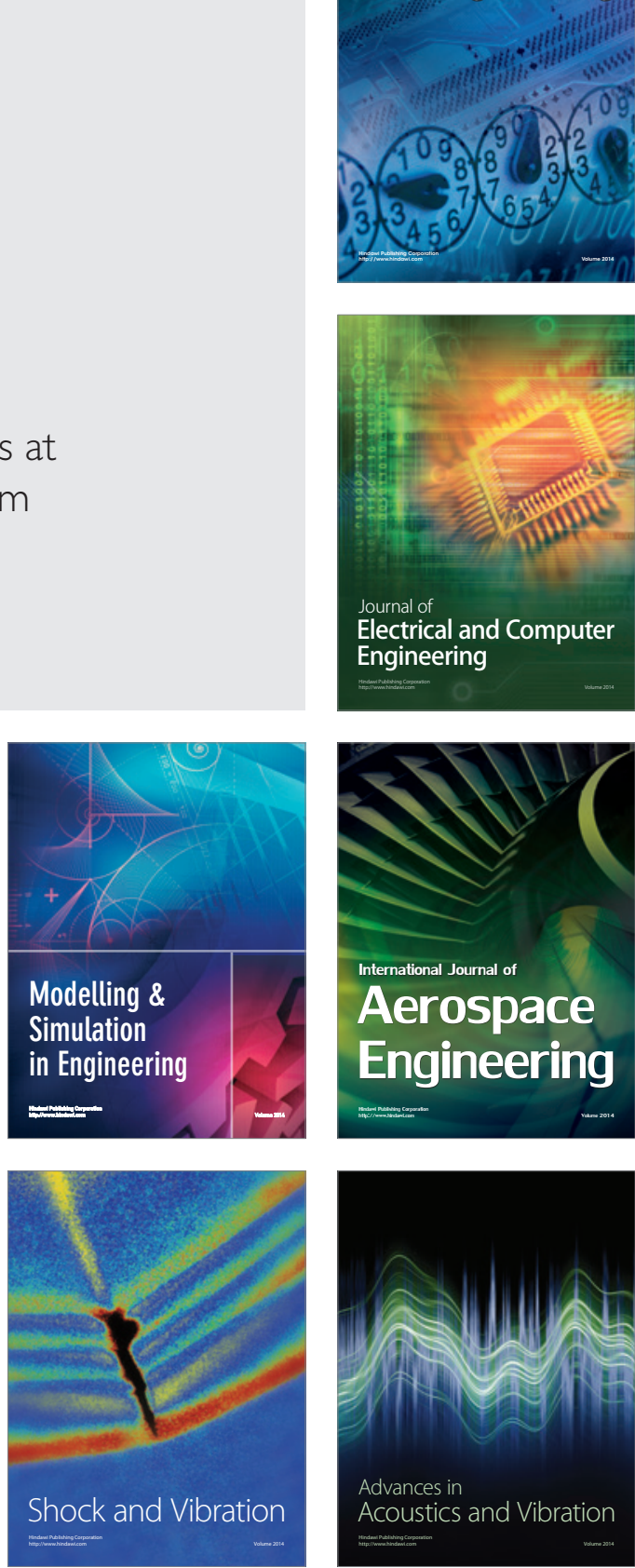